\section{Increased Coagulation With Aging: Importance of Homocysteine and Vitamin B12}

\section{To the Editor:}

Ochi et al $^{1}$ draw attention to an important issue in the management of elderly patients. Increased coagulation is an issue not only for deep vein thrombosis, but perhaps particularly for patients with atrial fibrillation. An issue not mentioned in either their paper or the accompanying editorial is the marked increase in the prevalence of hyperhomocysteinemia with aging. This clotting factor quadruples the risk of stroke in atrial fibrillation ${ }^{2}$ and is particularly important because it is usually treatable.

Deficiency of folic acid and metabolic B12 deficiency are treatable causes of hyperhomocysteinemia; in countries without folate fortification metabolic B12 deficiency is particularly important, because it is often missed. ${ }^{3}$ A serum total B12 level in the "normal" range (often $\sim 160-600 \mathrm{pmol} / \mathrm{L}$ ) does not equate with adequacy of functional B12, because only a small and variable fraction of $\mathrm{B} 12$ is active. To be confident that the serum total B12 excludes metabolic B12 deficiency, the serum B12 needs to be $>400 \mathrm{pmol} / \mathrm{L}$. To confirm adequacy of B12 function in patients with low normal serum total B12 it is necessary to measure the holotranscobalamin, or methylmalonic acid, level, which is elevated with B12 deficiency. In folate-replete patients, measuring the total homocysteine (tHcy) is a surrogate for methylmalonic acid.

Approximately $20 \%$ of elderly patients have B12 deficiency, and it is more common in vascular patients. Among patients attending my stroke prevention clinic, metabolic B12 deficiency is present in $10 \%$ of patients below age 50 , and $30 \%$ of patients aged 71 or higher. 4

It is becoming apparent that in early randomized trials of B vitamin therapy to lower homocysteine, harm from cyanide (as thiocyanate) from cyanocobalamin among study participants with impaired renal function cancelled out the benefit among participants with normal renal function. ${ }^{5}$ We should be using methylcobalamin.

The marked decline in renal function with age (Figure), ${ }^{6,7}$ combined with increased prevalence of metabolic B12 deficiency, results in high levels of tHcy in $40 \%$ of patients aged 80 or higher attending my stroke prevention clinic (Figure). Taken together with the marked increase in atrial fibrillation with age, these factors combine to make a perfect storm. All patients with atrial fibrillation should have their serum B12 and tHcy levels measured, and if they have metabolic B12 deficiency, they should be treated with methylcobalamin.

\section{References}

1. Ochi A, Adachi T, Inokuchi K, Ogawa K, Nakamura Y, Chiba $\mathrm{Y}$, et al. Effects of aging on the coagulation fibrinolytic system in outpatients of the cardiovascular department. Circ J 2016; 80: $2133-2140$.
2. Poli D, Antonucci E, Cecchi E, Marcucci R, Liotta AA, Cellai $\mathrm{AP}$, et al. Culprit factors for the failure of well-conducted warfarin therapy to prevent ischemic events in patients with atrial fibrillation: The role of homocysteine. Stroke 2005; 36: 2159-2163.

3. Spence JD. Metabolic vitamin B12 deficiency: A missed opportunity to prevent dementia and stroke. Nutr Res 2016; 36: 109-116.

4. Spence JD. Nutrition and stroke prevention. Stroke 2006; 37: $2430-2435$.

5. Spence JD. Homocysteine lowering for stroke prevention: Unravelling the complexity of the evidence. Int J Stroke 2016; 11: $744-747$.

6. Spence JD, Urquhart BL, Bang H. Effect of renal impairment on atherosclerosis: Only partially mediated by homocysteine. Nephrol Dial Transplant 2016; 31: 937-944.

7. Spence D. Mechanisms of thrombogenesis in atrial fibrillation. Lancet 2009; 373: 1006.

\section{J. David Spence, MD}

Stroke Prevention \& Atherosclerosis Research Centre, Robarts Research Institute, Western University,

London, ON, Canada

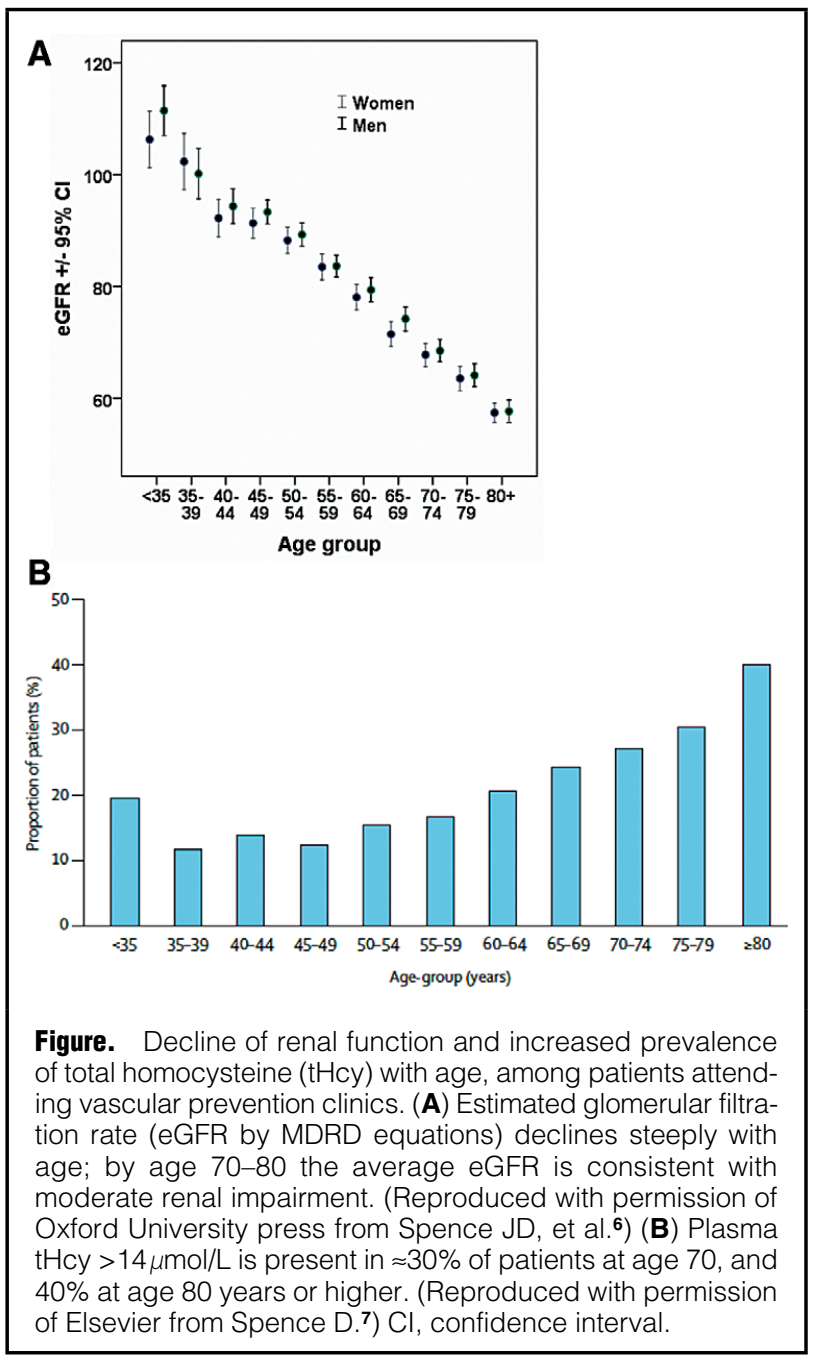

Received October 5, 2016; accepted October 5, 2016; released online December 16, 2016

Mailing address: J. David Spence, MD, FRCPC, FAHA, Stroke Prevention \& Atherosclerosis Research Centre, Robarts Research Institute, Western University, 1400 Western Road, London, ON, Canada N6G 2V4. E-mail: dspence@robarts.ca

ISSN-1346-9843 All rights are reserved to the Japanese Circulation Society. For permissions, please e-mail: cj@j-circ.or.jp 